\title{
Special issue on SIASP at ICDM 2010
}

\author{
Hakim Hacid · Tetsuya Yoshida • Cécile Favre
}

Published online: 3 March 2013

(C) Springer Science+Business Media New York 2013

With the emergence of Web 2.0, the user became the heart of various technologies that compose this new era such as mashups, collaborative environments, social networks, etc. The main added ingredient is certainly the social dimension with the aim of linking users together to facilitate their interaction and make it richer and more productive. The social Web is increasingly becoming the most interesting part of the Web and is at the point of challenging well established Web players such as the traditional search engines, e.g., Google. This is a huge step forward from a user perspective but also opens up great prospects for research in an environment that becomes increasingly complex, less structured and more hostile considering the great mass of knowledge generally hidden from the user.

Social networks are the focus of most of the work being done around the social Web. Works in this area address in particular the structural properties, e.g., the strength of social connections, characterization of key players, etc. Apart from social networks, the social dimension may be found in other forms and other locations on the Web: social media such as YouTube and Flickr, social news like Digg or Twitter, or social bookmarking as Delicious (del.icio.us). All these forms constitute a huge container of social information with knowledge that may be useful to the user. This knowledge may be leveraged by, e.g., offering new value added services exploiting that knowledge, which otherwise is very poorly exploited by users and service providers today.

H. Hacid $(\bowtie)$

Bell Labs France, Centre de Villarceaux Route de Villejust, 91620, Nozay, France

e-mail: hakim.hacid@alcatel-lucent.com

T. Yoshida

Graduate School of Information Science and Technology, Hokkaido University,

N-14 W-9, Sapporo 060-0814, Japan

e-mail:yoshida@meme.hokudai.ac.jp

C. Favre

Université Lumière Lyon 2, 5 avenue Pierre Mendès-France, 69676 Bron Cedex, France

e-mail: cecile.favre@univ-lyon2.fr 
This Special Issue contains a selection of the four best papers presented at the IEEE workshop on Social Interactions Analysis and Services Providers held in conjunction with IEEE ICDM 2010 in Sydney, Australia. The workshop aimed to bring together researchers from both academia and industry around issues related to the social interactions analysis through mining social data for providing users added value services. On the other hand, it is necessary to study and understand how services providers could leverage this huge mass of hidden knowledge for, e.g., business purposes, an issue facing all services providers in this area. Concretely, this includes confronting ideas in order to have a clearer view of the elements that surround this new phenomenon, to build a clear overview of advances in the various tracks related to the new Web era and then examining the scientific and industrial short, medium and long terms to rise around this hot topic. Some of the connected topics to the workshop include:

- Knowledge discovery from social data;

- Social networks (personal/professional) analysis;

- Social analysis for content and services personalization;

- Social interactions analysis for information retrieval and filtering;

- Social recommender systems;

- Community extraction and analysis;

- Privacy and social interactions analysis;

- New challenges and applications.

The workshop has attracted 14 papers directly submitted to the workshop and 12 papers received from the main conference. Only 12 papers have been accepted (eight from the workshop and four from the conference). Papers went through a rigorous review process. We did our best to get at least three reviews per paper. The workshop consisted of a series of presentation for the 12 accepted papers as well as a keynote talk about social networks analysis. At the end, the four best papers have been selected to be included in this special issue. These papers have been extended and went through a tough reviewing process which took more than one year and a half. We believe that now the papers are of a very good quality and satisfy the journal selection criteria. Hereafter is a brief description of the content of the selected papers:

In the first paper, entitled "Meme Ranking to Maximize Posts Virality in Microblogging Platforms", the author discuss issues related to content ranking for virality maximization. The paper introduces the meme ranking problem and formalizes it as the problem of selecting which $\mathrm{k}$ memes to show to users when they $\log$ into the system. The paper shows that not only exact solutions are unfeasible, but also approximated solutions are prohibitive to be adopted in an on-line setting. Authors devise a set of heuristics and compare them through an extensive simulation based on the real-world Yahoo! Meme social graph.

The second paper, entitled "Catching a Viral Video", discusses about content sharing on social networking sites and the effect on content popularity. The paper seeks a better understanding of viral videos on YouTube by an analysis of the "sharing" action and its relationship to video popularity using millions of YouTube videos. Authors found that viewership patterns of highly social videos are very different from less social videos. They also found that not all highly social videos become popular, and not all popular videos are highly social. A method has 
been developed for ranking blogs and websites on their ability to spread viral videos.

The third paper, entitled "Tag Recommendation by Machine Learning with Textual and Social Features", highlights a work on tags recommendation on social networking websites using machine learning techniques in a highly noisy context. Authors propose several novel features of tags for machine learning, called social features, as well as textual features to address this problem.

Finally, the last paper, entitled "Toward Finding Hidden Communities based on User Profile", considers the community detection problem from a partially observable network structure where some edges are not observable. The paper proposes to utilize a graph structure (called a profile graph) which is constructed via the profile data, and proposes a simple model to utilize both the observed connectivity relation and the profile graph. Furthermore, instead of a hierarchical approach, based on the modularity matrix of the network structure, an embedding approach which utilizes the regularization via the profile graph is used.

We would like to thank lots of people who helped and supported us for making this happen. Special thanks go to the journal Editor-in-Chief Prof. Zbigniew Ras for accepting our proposal to prepare this special issue. Many thanks to the IEEE ICDM workshop chairs who gave us this opportunity to set up the workshop and their advices and support during the whole organization period. Many thanks also to all the authors who submitted their papers to the workshop. Finally, special thanks go to all the PC members: David Carmel (IBM Research, Israel), Aiyou Chen (Alcatel-Lucent Bell Labs, USA), Ludovic Denoyer (University of Paris 6, France), Paul El-Khoury (SAP Research, France), Amol Ghoting (IBM T. J. Watson Research, USA), Laurent Gomez (SAP Research, France), Fabrice Guillet (Université de Nantes, France), Makoto Haraguchi (Hokkaido University, Japan), Silvija Kokalj (INRIA Saclay, France), Yiannis Kompatsiaris (Informatics and Telematics Institute, Greece), David Konopnicki (IBM Research, Israel), Yosi Mass (IBM Research, Israel), Mikolaj Morzy (Poznan University of Technology, Poland), Victor Muntés Mulero (Universitat Politècnica de Catalunya, Spain), Yoshiaki Okubo (Hokkaido University, Japan), Helen Paik (University of New South Wales, Australia), Sherif Sakr (University of New South Wales, Australia), Nisheeth Shrivastava (Alcatel-Lucent Bell Labs, India), Emina Soljanin (AlcatelLucent Bell Labs, USA), Julia Stoyanovich (University of Pennsylvania, USA), Aixin Sun (Nanyang Technological University, Singapore), Athena Vakali (Aristotle University of Thessaloniki, Greece), Eirini Giannakidou (Aristotle University of Thessaloniki, Greece), Christos Zigkolis (Aristotle University of Thessaloniki, Greece) who have accepted to share their experience by evaluating the papers, took the necessary time to process the papers, and for sending their reviews on time. We are grateful for their great support and help. 